Diese Befürchtung ist übertrieben. Schon vor vielen Jahren sah ich einen Mann, der den Vogel im Fluge traf und nur ein Auge besass mit einer kurzen, linienförmigen Pupille, infolge von doppelter Einheilung der Regenbogenhaut in eine Hornhautnarbe.

Seit einer Reihe von Jahren habe ich an sehenden Augen mit Hornhautflecken vor der Pupille die Sehkraft durch Schwarzfärbung des Flecks verbessert, und möchte mir erlauben, einen besonders ermuthigenden Fall kurz mitzutheilen.

Der 26jährige Arbeiter A. K. leidet seit 2 Jahren an Entzündung beider Augen. Beiderseits besteht eine kreideweisse (Blei-)Trübung der Hornhaut, welche die Pupille grösstentheils deckt, mit Thränen und Lichtscheu.

Rechts SnCC in $15^{\prime}$ kaum, $S n 4$ in $6^{\prime \prime}(S=1 / 10)$. Links Finger 10', Sn 16 in $6^{\prime \prime}(S<1 / 20)$. Augengrundbild unklar.

Die Behandlung mit Chlorumschlägen, Einträufelung von Atropin nebst Cocain u. s. w. bewirkte keine Besserung. Deshalb erfolgte am 12. März 1891 die Aufnahme und am folgenden Tage, unter Cocain, die Schwarzfärbung des rechten Flecks, mittels schräger Stichelung.

Am 31. März 1891 vollführte, wälrend meiner Reise, mein erster Assistent, Herr Dr. Perles, die gleiche Operation am linken Auge. Der Kranke wurde bald danach in reizlosem Zustand (mit $S=1 / 2$ rechts, $1 / 3$ links) entlassen und konnte wieder die Arbeit aufnehmen. Nur am Feuer ist er noch behindert.

Am 10. Mai 1891 erkennt jedes Auge für sieh (das linke mit $+20^{\prime \prime}$ ) SnXX: 15', von XV einzelne Buchstaben und liest $1^{1 / 2}$ in $10^{\prime \prime}$. $(S>3 / 4)$.
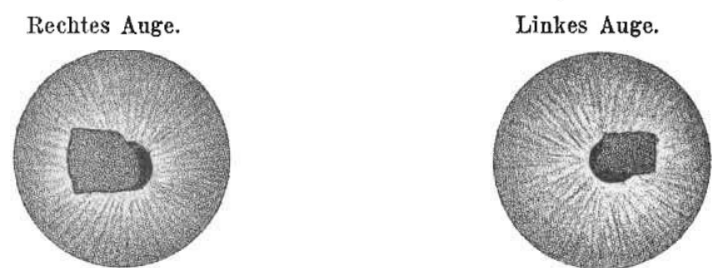

Die Figur zeigt übersichtlich das Verhältniss des Schwarzflecks zur Pupille. Es ist unmöglich, unter diesen Verhältnissen durch irgend eine Art von Pupillenbildung eine solcheVerbesserung der Sehkraft zu erzielen.

\title{
II. Literatur.
}

A) Die alten Griechen färbten Weissflecke der Hornhaut, wie die Schuster Naturleder schwärzen. Getrocknetes Pulver von lange auf bewahrten Galläpfeln wurde mit heisser Sonde auf den Fleck eingerieben und danach in Wasser gelöster Chalkanthos, d. i. schwefelsaures Kupfer, welches schwefelsaures Eisen enthält. Das Kupfer ist die Beize, das Eisensalz dringt ein und bildet im Gewebe selber den unlöslichen Niederschlag von gerb- (und gallus-) saurem Eisenoxyd, also von Tinte.

1. Galen, Band XII, S. 739. (Ausg. v. Kühn).

2. Aëtius, S. 131.

3. Alex. Trall, Band II, S. 51. (Ausg. v. Puschmann).

4. Theophanus Nonnus I. c. 68 , S. 246. (Ausg. v. Bernard).

Dies Verfahren war in Vergessenheit gerathen, bis nach de Wecker's Erfindung (1869) Anagnostakis wieder auf die Stelle aus Galen hinwies.

5. Contributions à l'histoire de la chirug. ocul. Athènes 1872, S. 41.

Ich selber sammelte die anderen Stellen und zeigte das Chemische des Verfahrens.

6. Wörterbuch der Augenheilk. 1887, S. 64.

\section{Hornhautfärbung gegen Pupillenbildung.}

\author{
Von Prof. Dr. J. Hirschberg.
}

I. Jedem Erfahrenen ist genügend bekannt, dass bei Hornhautflecken, welche nicht die ganze Pupille, sondern nur einen Theil derselben, wenngleich einen grösseren, verdecken, die Bildung einer künstlichen Pupille, durch Aus- oder Zerschneidung der Regenbogenhaut, wenig oder gar nicht zur Verbesserung der Sehkraft beiträgt.

Die Sehstörung beruht eben (zwar nicht ausschliesslich, aber doch hauptsächlich) auf der Lichtzerstreuung, die von dem halbdurchscheinenden Fleck ausgeht. Schwarzfärbung der Hornhautnarbe verbessert das Sehen. Das ist schon seit nahezu 20 Jahren bekannt.

Nichtsdestoweniger wird die Färbung von Hornhautnarben ohne Pupillenbildung, soviel ich gesehen und gelesen, im ganzen nur selten ausgeführt: einmal, weil in den gebräuchlichen Lehrbüchern ein gutes Verfahren, um in einer einzigen Sitzung den weissen Fleck dauernd kohlschwarz zu färben, nicht mitgetheilt wird $\left.;^{1}\right)$ sodann, weil man befürchtet, das zurückbleibende $S \mathrm{ehloch}$ könnte $\mathrm{zu}$ schmal ausfallen.

1) Die neuesten Lehrbücher, wie das vorzügliche von SchmidtRimpler (V. Auf. 1891, S. 468), sprechen nur von der kosmetischen Tätowirung, ohne Fixation des Augapfels, und empfehlen eine Reihe von
7. C. Bl. f. A. 1887, S. 69

B) 1869, durch eine Frage des bei ihm studirenden Abadie angeregt, vollführte de Wecker zuerst die Tätowirung eines Weissflecks der Hornhaut mittels einer Nadel und chinesischer Tusche. Er hat auch gelehrt, durch Färbung halb durchscheinender Flecke in der Hornhantmitte, nach der Pupillenbildung, die Sehkraft zu verbessern und nach Staphylomabtragung die Bindehaut zu tätowiren.

8. de Wecker, Union médicale No. 27.

9. derselbe, Wien. med. W. 1871.

10. derselbe, Arch. d. Augenheilk. von Knapp 1872, II, 2, 84.

11. derselbe, Thérapeutique oculaire 1878, S. 207-210.

12. derselbe, Chirurgie oculaire, 1879, S. 181-183.

13. derselbe, Traité complet d'ophth. 1886, II, S. 184 bis 185.

Sitzungen. Aehnlich Berry (Edinburgh 1889, S. 614) und Galezowski (III. Aufl., Paris 1888, S. 297). Diese Beispiele mögen genügen.

Mein eignes Verfahren habe ich im Centralbl. f. Aug. 1887, S. 69 geschildert. Ich lasse den Kranken sitzen, cocainisire gut, fixire den Augapfel, indem ich oben, mit Gummipincette, die Bindehaut fasse; stichele schräg und vielfach mit 8 fachem Nadelbündel und reibe die chinesische Tusche kräftig mit dem Finger ein. Eine Sitzung genügt. Reizung erfolgt niemals. Das Tuschstück wird vorher in einem Glasgefäss durch trockne Hitze $\left(130^{\circ}\right.$ C) sterilisirt, zum Gebrauch mit gekochter Sublimatlösung $(1: 5000)$ verrieben, das Nadelbündel unmittelbar vor der Operation ausgekocht. 
Das Verfahreu wurde vou Vielen geübt und namentlich durch Taylor verbessert, der die Tusche auf deu Fleck strich (ebenso wie Bader), eiu Büudel vou 4 Nadelu zur Stichelung benutzte, sowie einen Spatel zum Eiureiben. Levis hat vorgeschlageu, auch die gesunde Horuhant zu tätowiren: bei Mydriasis, ferner die Mitte des Keratocouns u. s. w. Völckers hat gezeigt, dass Horuhantuarbeu durch Tätowirung widerstandsfähiger uud weuiger geneigt zu Eutzüuduugsrückfälleu gemacht werden. Ich selber verdeckte (nach dem Vorgaug vou Reuss, 41) deu hässlicheu Weissstar des stockbliuden Anges durch Schwarzfärbung der Mitte der gesundeu Horuhaut und verbesserte die Sehkraft bei centraleu Horuhautfeckeu ohue Iridectomie, durch Tätowirung des Fleckes.

14. v. Reuss, Wiener med. Presse 1870 , S. 975.

15. Rydel, Przeglad Lekarski 1871, No. 16 u. 17. (Auch gegeu Lichtdiffusion).

16. Schmidt-Rimpler, Nagel's J. B. 1872, S. 273. S. 86 .

17. Keller, Sitz.-Ber. d. V. d. Aerzte i. Steiermark, 1872,

18. Bergh, Deutsche Klinik 1872, No. 6.

19. Talko, Klin. Mouatsbl. f. A., X, 265.

20. Woiu ow, Sitz. B. d. G. russ. Aerzte 1872, No. 13.

21. Ravà, Del Tatuaggio della coruea, Sassari 1872

22. Levis, Philad. med. Times 1872, 5. Oct.

23. Duunage, Med. Times a. G., B. 44, S. 294.

24. Ticehurst, Laucet I, 610, 1872.

25. Taylor, Brit. med. J., 7. Sept. 1872.

26. War lomout, Auual. d'Oc. 67, S. 75, 1872.

27. v. Reuss, Wieu. med. Presse 1873 , S. 6.

28. Adler, Wieu. med. W. 1873, S. 202-203, und Bericht (1874)

29. Berger (v. Rothmu d), Blätter f. Heilw. 1, 2.

30. v. Hasuer, Beitr. z. Phys. u. Path. d. A., S. 80 fgd.

31. Pouti, Anuali di ottalm. II 507 fgd. und III 169, (mit Wecker)

32. Thoms ou, Trausact. Amer. O. S., S. 86.

33. Williams, Bost. med. a. surg. J., 3. July 1873.

34. Archer, Arch. f. 0. XX, 1, 225 fgd.

35. Kleiu, Wieuer med. Presse 1874, No. 38, 39.

36. Oeffinger, Aerztl. Mitth. ans Badeu 1874, No. 10 .

37. Osio, Cronica oftalm. Sept. 1874.

38. Poucet, Gazette des hôpitaux, 1876, No. 28 u. Progrès méd. S. 210.

(Die Tusche fiudet sich in deu untersteu Lageu des Epithels, sowie in den Zellkeruen uud Lymphkörperchen der vordersten Schichteu des eigentlichen Horuhautgewebes).

39. Holm, Dissert., Kiel (Völckers) u. Arch. f. exper. Pathol. VI, S. 256. (Der Farbstoff wird von deu Eudothelien der Gefässe aufgenommeu, beim Kaniuchenversuch. - Völckers will durch Tätowirung eine Verödung der uengebildeteı Gefässe erzielen und so die Rückfälle der Entzündung abschueiden).

40. Hock, Arch. f. Aug. u. 0. V, 1, 90 fgd.

41. v. Reuss, Kliu. Mouatsbl. f. A. 1876, S. $139 \mathrm{fgd}$.

42. Klein, M. des Wien. med. Doct. Colleg. II, 109.

43. Pietrowski, Milit. med. J. 1877, Febr. (Russisch).

44. Browicz, Arch. f. O. XXIII, 3, 312 fgd.

(Histolog. Uutersuchung eines Anges, das 2 Jahre zuvor, wegeu Horuhautuarbe nach Geschwür, tätowirt worden. An der Narbenstelle fehlt die Bowmau'sche Schicht. Unter dem Epithel bestand die Horuhant ans feiufibrillärem Gewebe, mit zahlreicheu Blntgefässeu. Die Farbstoffköruer lagen in platte Zelleu eingeschlossen, theils in deu Spaltränmen als grössere Schollen, theils in den Wäudeu der Blntgefässe als Köruer. Die Farbstoffanhäufuug uahm die vordere Hälfte der Horuhantdicke ein).

45. Pauas, Gaz. des hôp. 1878. 85 .

(Sah nach der Operation Iridocyclitis, auch sympathische!)

46. Hirschberg, Auatom. u. pr. Bemerk. zur . . . Horuhautfärbung, A. f. O. XXVIII, 1, 269 fgd., mit Abbild., 1882.

(Der erblindete, ektatische Augapfel hatte $1 \mathrm{Jahr}$ nach der Färbung entferut werden müssen. Das Epithel ist frei vou der Färbung; das giebt den schönen Glauz des Flecks. Das Pigment sitzt iu deu vorderen Schichten der uarbigen Horuhaut bis zu $1 / 4$ ihrer Dicke, z. Th. in Zelleu, meist ausserhalb derselben; anch in den Wandnugen neugebildeter Blntgefässe).

47. Kotelmaun, Der zoolog. Garteu 1884, XXV, No. 9.

(Tätowirnug bei einem Lämmergeier. - Bei Pferden haben es Zuhörer vou mir ausgeführt).

48. Mayerhauseu, C. Bl. f. A. 1885 , S. 230.

49. Hirschberg, C. Bl. f. A. 1887 , S. 69

50. L. Kugel, A.f. 0. XXXVI, 2, 1890.

III. Obwohl also gegen fünfzig Einzelmittheilungen vorliegen, sind doch die üblichen Lehrbücher uuvollständig iu Beziehung anf deu vorliegendeu Gegenstand. Folgeude Hauptsätze lehrt die Erfahrung:

1. Die Tätowirung der Hornhaut verbessert entweder uur das Ausseheu oder auch uoch dazu das Seheu.

2. Bei vollständiger Weissuarbe der Horuhaut (ohue Lichtscheiu; oder mit Lichtscheiu, aber ohue Vorderkammer) färbt man anf die Horuhantmitte eiue Pupille auf und ahmt in der Peripherie durch punktförmige Stichelung die Iris nach.

3. Bei vollständigem Weissstar ohue Lichtscheiu färbt man in der gesunden Horuhaut eine grosse ceutrale Pupille.

4. Eine unvollständige, aber grosse Weissuarbe der Horuhaut wird iu ihrer ganzen Ausdehunug schwarz gefärbt.

5. "Eutspauuende" Pupillenbildung ist i. A. unuöthig zur Färbnug und uur daun anszuführen, weun sie eineu Selbstzweck hat, sei es Drucksteigerung herabzusetzen, sei es eine verschlosseue $\mathrm{Pu}$ pille wieder zu öffuen.

6. Ist eiu, wenn anch schmaler Bezirk der natürlicheu Pupille frei, so wird bessere Sehkraft erzielt durch einfache Färbuug, ohue Iridectomie.

7. Die Horuhautfärbung ist gauz gefahrlos bei strenger Asepsie; sie macht niemals Reizung und kanu anch anf gute Augeu angeweudet werden, z. B. mit kleiuem Hornhautfleck im Pupilleubereich; oder mit Horuhautkegel, uach dem Breuneu; oder mit Schichtstar, uach der Iridectomie.

8. Die Stichelung soll schräg seiu, besouders bei düuuer Horu- 